RESEARCH ARTICLE

\title{
Species composition of mosquitoes associated with a livestock field station
}

\author{
Hansani S. S. Daluwaththa ${ }^{1}$, S. H. P. Parakrama Karunaratne ${ }^{1}$ and W.A. Priyanka P. de Silva ${ }^{1, *}$
}

${ }^{1}$ Department of Zoology, Faculty of Science, University of Peradeniya, Peradeniya, Sri Lanka

Received:28/05/2018; Accepted:07/08/2018

\begin{abstract}
Blood feeding nature of mosquitoes associated with livestock field stations are crucial in understanding the vectorborne disease dynamics. We investigated the species composition, abundance and blood feeding pattern of mosquitoes at a livestock field station in Uda Peradeniya, Sri Lanka. Monthly samplings were done from six animal units (cattle, swine, duck, sheep, rabbit, and goat) from December 2016 to July 2017 using hand held aspirators, UV light traps and CDC miniature light traps. Blood sources of the mosquitoes were confirmed by PCR assays. Majority of the mosquitoes $(82.3 \%)$ were belonged to the Genus Culex followed by Armigeres (7.31\%), Aedes (6.70\%) and Anopheles (3.65\%). Culex fuscocephala (26.82\%) was the most abundant species while Aedes albopictus $(0.30 \%)$ and Anopheles aitkenii $(0.30 \%)$ were the least. The highest mosquito abundance was reported from cattle unit $(86.9 \%)$ followed by swine $(12.2$ $\%)$ and duck (0.91\%) units. The highest mosquito abundance and diversity were reported in December (35.97\%). No association were reported between climatic variables and mosquito abundance $(p>0.05)$. Mixed blood meals were not reported and mosquitoes of cattle and swine units were identified with $100 \%$ cattle and swine blood, respectively. The generalized feeding pattern of the identified mosquitoes suggests the potential vector status of infectious diseases.
\end{abstract}

Keywords: Mosquito abundance, Livestock field stations, Species composition, Host choice, Blood meal analysis, Feeding pattern.

\section{INTRODUCTION}

Blood feeding nature of mosquitoes is shaped by many biological and ecological factors (Takken and Verhulst, 2013). The range of the host species of a mosquito is mainly determined by the fitness advantage it gains by being a specialized feeder or a generalist feeder (Pyke et al., 1977; Abrams, 2006; Takken and Verhulst, 2013). Even though specialist feeding mosquito species are critical in transmitting many enzootic diseases within host populations, majority of generalist feeding mosquito species are likely to be bridge vectors in transmitting mosquito borne pathogens between populations (Gingrich and Williams, 2005). For instance, several Culex species have shown an opportunistic generalized feeding pattern by taking blood meals from a wide variety of avian and mammalian hosts (Tempelis and Washino, 1967; Tempelis et al., 1976; Garcia-Rejon et al., 2010) and acted as bridge vectors of many mosquito borne diseases including West Nile virus, Filariasis, Japanese encephalitis (Thiemann et al., 2011; Bhattacharya et al., 2016).

One of the contributory factors for emerging and reemerging of zoonotic diseases is the animal domestication and livestock farming (Lima et al., 2014). Increased risk of introducing new pathogens to human populations through mosquito species with generalized feeding patterns has become a crucial factor in controlling zoonotic diseases. Animal domestication and livestock farming have given ample opportunities for these mosquitoes to go for a number of host species to obtain successful blood meals and increasing their foraging efficiency (Santos and Borges, 2015). Different domestic animals live in the same environment provide a dynamic environment for diverse mosquito species that contributes to inter and intra species transmission of pathogens.

According to WHO (2017) reports, Livestock are useful in diverting mosquitoes that attracted to humans. Thus, having livestock close to human settlements are important in reducing human bites of mosquitoes. However, it has been shown that livestock indirectly attracts more mosquitoes to human settlements through host derived signals. Thus having a livestock station within a human settlement increases the attraction of zoophilic mosquitoes and the human biting rate (Reviewed in Seyoum et al., 2002). Changing the vegetation and the natural environmental conditions by establishing livestock stations introduces new mosquito breeding sites. Such habitat changes subsequently increase the vector population and the risk of disease transmission leading to many epidemics (Lehane, 2005; Santos and Borges, 2015). The livestock have, thus, indirectly shaped up the evolution of generalized feeding behavior of mosquitoes that live in human settlements. Generalist blood feeding pattern of these mosquitoes, therefore, is essential in understanding and describing zoonotic diseases that can be transmitted to human population as well as to other vertebrates in livestock. Few such studies have been carried out to understand the possible risks of emergence of zoonotic diseases associated with livestock field stations. The objective of the present study was to determine the species composition of blood feeding mosquitoes in a livestock station and to understand their feeding pattern and host choice. 


\section{MATERIALS AND METHODS}

\section{Adult and larval mosquito collection and identification}

Mosquitoes were collected from six animal units i.e. Cattle unit, Swine unit, Duck unit, Sheep Unit, Rabbit-Quail Unit and Goat-Sheep Unit of the livestock field station, Mawela, Uda Peradeniya ( $7^{\circ} 15^{\prime} 15^{\prime \prime} \mathrm{N} ; 80^{\circ} 35^{\prime} 48^{\prime \prime}$ E; 598 $\mathrm{m}$ above sea level), Sri Lanka (Figure 1) during the period of December 2016 to July 2017. Monthly collections were done in first week of every month throughout the study period from each animal unit from 1800 hours to 2100 hours using hand held mechanical aspirators (Hausherr's machine works, USA), heavy duty hand-held dc/vacuum aspirator (BioQuip Products, Rancho Dominguez, CA, USA), CDC miniature light traps (BioQuip Products, Rancho Dominguez, CA, USA) and UV traps (BioQuip Products, Rancho Dominguez, CA, USA).

Aspirators were used to collect mosquitoes directly from the animal body surfaces and from other resting surfaces at each animal unit. UV traps and CDC miniature light traps were set up at six animal units for three hours per day to collect blood fed mosquitoes that attracted to those traps. Collected mosquitoes were transferred to small vials kept in a cooler to delay the digestion of blood meals and transported to the laboratory where they were stored at $-20^{\circ} \mathrm{C}$ until further use.

Larvae were collected from the water samples obtained from all available breeding sites (drainage systems, containers, tires and damaged PVC pipes) of the Livestock station. They were brought to the laboratory and reared into adults with fish food as the food source. Pupae were transferred to mosquito rearing cages for adult emergence (Gaffigan and Pecor, 1997). Adult mosquitoes were examined under an AmScope SM-2B Professional Binocular Stereo Zoom Microscope and identified using available taxonomic keys (Barraud, 1934; Amerasinghe, 1990 and 1995). These mosquitoes were separated according to the visual blood meals in their abdomen fully blood fed, partially blood fed and non-blood fed individuals.

\section{Blood meal analysis}

A total of randomly selected 75 blood fed mosquitoes were analyzed to identify the host type. Each engorged abdomen was extracted following the Livak method (Livak, 1984). Cytochrome $\mathrm{b}$ gene was amplified using four mammalspecific forward primers [i.e. Human 741F, Cow 121F, Goat 894F (common for both goat and sheep) and Pig $573 F$ ] and one universal reverse primer (UNREV 1025), and a set of avian primers (forward and reverse Avian "a") following Kent and Nooris, 2005 and Molaei et al., 2006 (Table 1).

Each PCR amplification was done for a total of 25.0 $\mu \mathrm{L}$ solution that included $5.0 \mathrm{uL}$ of extracted blood DNA, 13.0 $\mu \mathrm{L}$ of double distilled water, 3.0 $\mu \mathrm{L}$ of KapaTaq Buffer, $1.5 \mu \mathrm{L}$ of $\mathrm{Mgcl}_{2}, 0.25 \mu \mathrm{L}$ of dNTPS, $0.25 \mu \mathrm{L}$ of Taq polymerase and $1 \mu \mathrm{L}$ each of forward and reverse primers. For mammalian primers, initial denaturation of five minutes at $95^{\circ} \mathrm{C}$ was followed by 35 cycles of denaturation at $95^{\circ} \mathrm{C}$ for one minute, hybridization at $58^{\circ} \mathrm{C}$ for one minute and extension at $72^{\circ} \mathrm{C}$ for one minute. Final extension step was done at $72^{\circ} \mathrm{C}$ for seven minutes.For avian primers, the thermal cycle with an initial denaturation of five minutes at $95^{\circ} \mathrm{C}$ was followed by 36 cycles of denaturation at $94^{\circ} \mathrm{C}$ for 30 seconds, hybridization at $60^{\circ} \mathrm{C}$ for 50 seconds and extension at $72^{\circ} \mathrm{C}$ for 40 seconds. Final extension was done

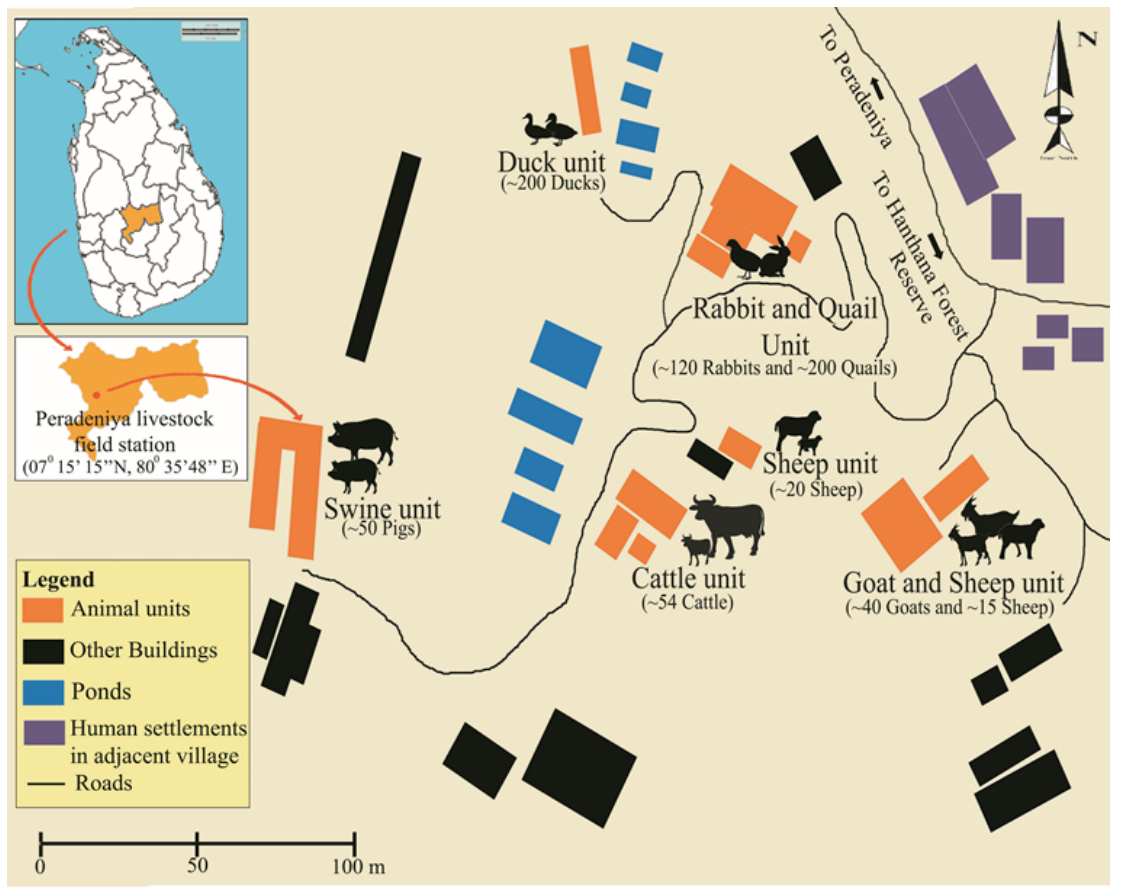

Figure 1: A map showing the livestock field station, Mawela, Udaperadeniya, Sri Lanka. 
Table 1: Primers sets used to amplify Cytochrome b gene of vertebrate blood meal (avian and mammal) in engorged mosquitoes.

\begin{tabular}{llc}
\hline \multicolumn{1}{c}{ Primer } & \multicolumn{1}{c}{$\mathbf{5}$ '- $\mathbf{3}$ ' sequence } & Product size (bp) \\
Human741F & GGCTTACTTCTCTTCATTCTCTCCT & 334 \\
Cow121F & CATCGGCACAAATTTAGTCG & 561 \\
Goat894F & CCTAATCTTAGTACTTGTACCCTTCCTC & 132 \\
Pig 573F & CCTCGCAGCCGTACATCTC & 453 \\
UNREV1025 & GGTTGTCCTCCAATTCATGTTA & - \\
Avian a for & GACTGTGACAAAATCCCNTTCCA & 508 \\
Avian a rev & GGTCTTCATCTYHGGYTTACAAGAC & - \\
\hline
\end{tabular}

at $72^{\circ} \mathrm{C}$ for seven minutes. Amplified DNA products were visualized by gel electrophoresis on Midori Green stained $1.5 \%$ agarose gels. Electrophoresis was conducted with Gene Ruler 100 base pair (bp) molecular mass marker.

\section{Data analysis}

Minitab Version 14 was used for data analysis. Significant tests were done using One-Way ANOVA to compare monthly abundance of mosquito species. Pearsoncorrelation coefficients were computed to determine the correlation between mosquito abundance and weather parameters. Dendrogram analysis with Bray Curtis Similarity test was done using Primer E6 software to compare similarities between animal units based on the mosquito species composition. Shannon-Weiner index and Evenness index were used to determine the diversity and evenness of blood feeding mosquito species. The total number of mosquitoes collected at each sampling were correlated with the weather variables i.e. temperature, rainfall, relative humidity and wind velocity which were obtained from the Natural Resources Management Center in Sarasavi Mawatha, Peradeniya, Sri Lanka to determine the association between climatic parameters and the mosquito abundance.

\section{RESULTS}

A total of 328 mosquitoes belonging to 4 genera and 17 species were collected (Table 2). The dominant mosquito genus in the livestock field station was Culex $(82.3 \%$; $\mathrm{n}=270)$ followed by Armigeres $(7.31 \% ; \mathrm{n}=24)$, Aedes $(6.70 \% ; \mathrm{n}=22)$ and Anopheles $(3.65 \% ; \mathrm{n}=12)$. Out of the 17 mosquito species, $10(58.8 \%)$ species belonged to the genus Culex and the most abundant mosquito species was Cx. fuscocephala which represented $26.82 \%(\mathrm{n}=88)$ of the collection. Aedes albopictus and Anopheles aitkenii were the least abundant mosquito species (Table 2).The highest mosquito abundance was recorded from the cattle unit $(86.89 \% ; \mathrm{n}=285)$ followed by the swine unit $(12.19 \%$; $\mathrm{n}$ $=40)$ and duck unit $(0.91 \% ; n=3)$. Four mosquito species (i.e. Cx. fuscocephala, Cx. gelidus, Cx. tritaeniorhynchus and $C x$. vishnui) were common to both cattle and swine units. Although the monthly abundance of $C x$. fuscocephala mosquitoes showed a significant difference in two units
$(\mathrm{F}=5.97 ; \mathrm{p}=0.028)$, that of other three species viz $C x$. vishnui $(\mathrm{F}=0.56 ; \mathrm{p}=0.466)$, Cx. gelidus $(\mathrm{F}=1.92 ; \mathrm{p}=$ $0.188)$ and $C x$. tritaeniorhynchus $(\mathrm{F}=3.92 ; \mathrm{p}=0.068) \mathrm{did}$ not show any significant difference. Only three mosquitoes i.e. two Cx. hutchinsoni and one Ae. albopictus, could be collected from the duck unit and these species could not be collected from anywhere else. No mosquitoes could be collected from the sheep unit, rabbit- quail unit and goatsheep unit.

Results of the blood meal diagnostic tests confirmed that the tested engorged females had taken blood meals only from the host type in the unit where they were collected (Figure 2). No mixed blood meals in the collected mosquitoes were identified. From the total of 75 mosquitoes 54 mosquitoes were diagnosed with the cattle blood meals. These mosquitoes belonged to Cx. fuscocephala (n $=5), C x$. pseudovishnui $(\mathrm{n}=5), C x$. gelidus $(\mathrm{n}=6), C x$. tritaeniorhynchus $(\mathrm{n}=6), C x$. vishnui $(\mathrm{n}=2), C x$. siteins $(\mathrm{n}=4), C x$. whitmorei $(\mathrm{n}=2)$, Culex unidentified sp. $(\mathrm{n}=$ 4), Ar. subalbatus $(\mathrm{n}=7)$, Armigeres unidentified sp. $(\mathrm{n}=$ 3), Ae. vittatus $(\mathrm{n}=7)$, An. jamesii $(\mathrm{n}=1)$, An. aitkenii $(\mathrm{n}=1)$ and Anopheles unidentified sp. $(\mathrm{n}=1)$. The rest 21 mosquitoes were diagnosed with swine blood meal and belonged to $C x$. fuscocephala $(\mathrm{n}=7), C x$. gelidus $(\mathrm{n}=5)$, $C x$. tritaeniorhynchus $(\mathrm{n}=2), C x$. vishnui $(\mathrm{n}=4)$ and $C x$. quinquefasciatus $(\mathrm{n}=3)$.

The Shannon-Wiener index for the diversity determination was ranged from 0.892 to 1.922 . The evenness values were found in the range from 0.214 to 0.308 . The cattle unit showed a relatively high species richness throughout the study period, however, the highest richness in this unit was reported in the month of December. The swine unit showed a moderate richness with a relatively high richness in the month of April. Mosquito abundance data showed monthly fluctuations and the highest mosquito abundance was recorded in the month of December. Some of the mosquito species were recorded throughout the study period, whereas some species were reported only in specific months (Table 3). 
Table 2: Species composition and abundance (ab.) of collected mosquitoes from the Udaperadeniya livestock field station.

\begin{tabular}{lcccccccc} 
& \multicolumn{2}{c}{ Cattle unit } & \multicolumn{2}{c}{ Swine unit } & \multicolumn{2}{c}{ Duck unit } & \multicolumn{2}{c}{ Total } \\
Species & $\mathbf{A b .}$ & $\mathbf{a b .} \%$ & $\mathbf{a b .}$ & $\mathbf{a b .} \%$ & $\mathbf{a b .}$ & $\mathbf{a b . \%}$ & $\mathbf{a b .}$ & ab. $\%$ \\
Cx. fuscocephala & 75 & 22.86 & 13 & 3.96 & 0 & 0.00 & 88 & 26.82 \\
Cx. gelidus & 40 & 12.19 & 15 & 4.57 & 0 & 0.00 & 55 & 16.76 \\
Cx. pseudovishnui & 43 & 13.10 & 0 & 0.00 & 0 & 0.00 & 43 & 13.10 \\
Cx. tritaeniorhynchus & 38 & 11.58 & 2 & 0.61 & 0 & 0.00 & 40 & 12.19 \\
Cx. vishnui & 9 & 2.74 & 4 & 1.21 & 0 & 0.00 & 13 & 3.96 \\
Cx. siteins & 6 & 1.82 & 0 & 0.00 & 0 & 0.00 & 6 & 1.82 \\
Cx. whitmorei & 2 & 0.61 & 0 & 0.00 & 0 & 0.00 & 2 & 0.61 \\
Cx. quinquefasciatus & 0 & 0.00 & 6 & 1.82 & 0 & 0.00 & 6 & 1.82 \\
Cx. hutchinsoni & 0 & 0.00 & 0 & 0.00 & 2 & 0.61 & 2 & 0.61 \\
Culex unidentified sp. & 15 & 4.57 & 0 & 0.00 & 0 & 0.00 & 15 & 4.57 \\
\hline Total & $\mathbf{2 2 8}$ & $\mathbf{6 9 . 5 1}$ & $\mathbf{4 0}$ & $\mathbf{1 2 . 1 9}$ & $\mathbf{2}$ & $\mathbf{0 . 6 1}$ & $\mathbf{2 7 0}$ & $\mathbf{8 2 . 3 1}$ \\
\hline Ae. vittatus & 21 & 6.40 & 0 & 0.00 & 0 & 0.00 & 21 & 6.40 \\
Ae. albopictus & 0 & 0.00 & 0 & 0.00 & 1 & 0.30 & 1 & 0.30 \\
\hline Total & $\mathbf{2 1}$ & $\mathbf{6 . 4 0}$ & $\mathbf{0}$ & $\mathbf{0 . 0 0}$ & $\mathbf{1}$ & $\mathbf{0 . 3 0}$ & $\mathbf{2 2}$ & $\mathbf{6 . 7 0}$ \\
\hline Ar. subalbatus & 13 & 3.96 & 0 & 0.00 & 0 & 0.00 & 13 & 3.96 \\
Armigere unidentified sp. & 11 & 3.35 & 0 & 0.00 & 0 & 0.00 & 11 & 3.35 \\
\hline Total & $\mathbf{2 4}$ & $\mathbf{7 . 3 1}$ & $\mathbf{0}$ & $\mathbf{0 . 0 0}$ & $\mathbf{0}$ & $\mathbf{0 . 0 0}$ & $\mathbf{2 4}$ & $\mathbf{7 . 3 1}$ \\
\hline An. jamesii & 4 & 1.21 & 0 & 0.00 & 0 & 0.00 & 4 & 1.21 \\
An. aitkenii & 1 & 0.30 & 0 & 0.00 & 0 & 0.00 & 1 & 0.30 \\
Anopheles unidentified sp. & 7 & 2.13 & 0 & 0.00 & 0 & 0.00 & 7 & 2.13 \\
\hline Total & $\mathbf{1 2}$ & $\mathbf{3 . 6 5}$ & $\mathbf{0}$ & $\mathbf{0 . 0 0}$ & $\mathbf{0}$ & $\mathbf{0 . 0 0}$ & $\mathbf{1 2}$ & $\mathbf{3 . 6 5}$ \\
\hline Total mosquitoes & $\mathbf{2 8 5}$ & $\mathbf{8 6 . 8 9}$ & $\mathbf{4 0}$ & $\mathbf{1 2 . 1 9}$ & $\mathbf{3}$ & $\mathbf{0 . 9 1}$ & $\mathbf{3 2 8}$ & $\mathbf{1 0 0 . 0 0}$ \\
\hline & & & & & & & &
\end{tabular}
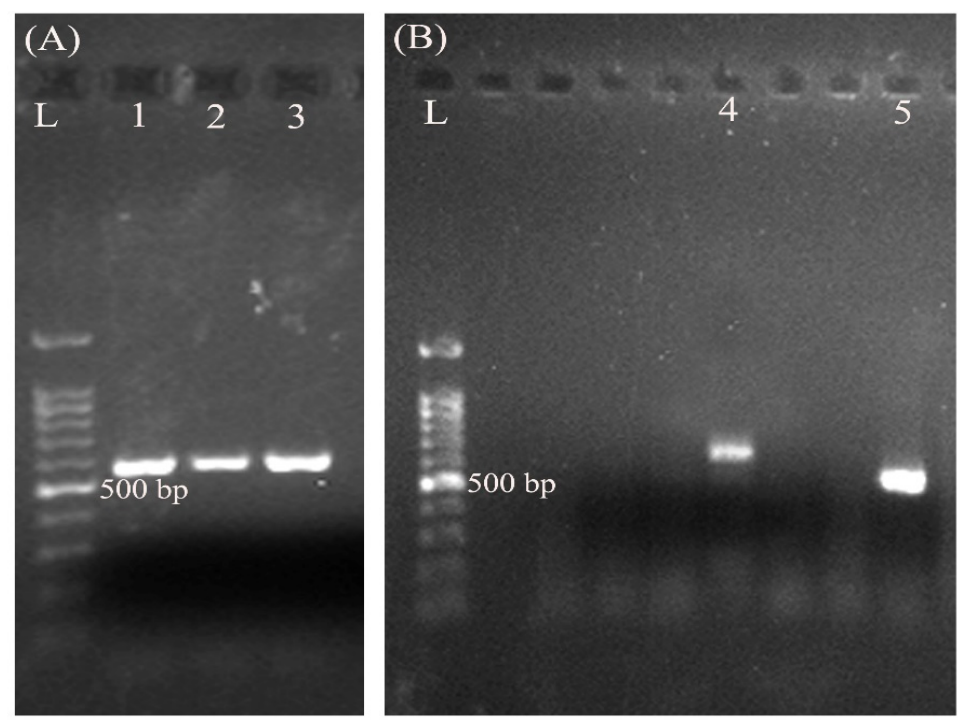

Figure 2: Gel images of extracted blood meals of individual mosquitoes collected from Livestock field station. DNA was extracted from the blood fed individuals. L, represented the DNA marker ladder. Identified blood meals of three individuals of Culex gelidus are indicated as 1,2,3 at 561bp (cattle blood) in the gel image "A". Identified blood meals of Culex tritaeniorhynchus (at 561 bp; cattle blood) and Culex quinquefasciatus (at 453 bp; swine blood) are indicated as 4 \& 5 respectively in the gel image "B". 
Table 3: Monthly abundance of mosquitoes captured in the livestock field station.

\begin{tabular}{lcccccccc}
\hline \multicolumn{1}{c}{ Mosquito species } & Dec. & Jan. & Feb. & Mar. & Apr. & May & June & July \\
\hline Cx. pseudovishnui & 40 & 0 & 1 & 0 & 0 & 2 & 0 & 0 \\
Cx. gelidus & 18 & 0 & 6 & 4 & 6 & 17 & 4 & 0 \\
Cx. fuscocephala & 12 & 0 & 8 & 6 & 14 & 25 & 23 & 0 \\
Cx. vishnui & 9 & 0 & 2 & 0 & 1 & 1 & 0 & 0 \\
Cx. tritaeniorhynchus & 12 & 0 & 1 & 5 & 2 & 17 & 3 & 0 \\
Cx. sitiens & 6 & 0 & 0 & 0 & 0 & 0 & 0 & 0 \\
Cx. whitmorei & 0 & 0 & 0 & 0 & 0 & 1 & 1 & 0 \\
Cx. quinquefasciatus & 0 & 0 & 0 & 0 & 4 & 2 & 0 & 0 \\
Cx. hutchinsoni & 2 & 0 & 0 & 0 & 0 & 0 & 0 & 0 \\
Culex unidentified sp. & 15 & 0 & 0 & 0 & 0 & 0 & 0 & 0 \\
Ae. vittatus & 0 & 0 & 1 & 19 & 1 & 0 & 0 & 0 \\
Ae. albopictus & 0 & 0 & 0 & 1 & 0 & 0 & 0 & 0 \\
Ar. subalbatus & 0 & 0 & 0 & 0 & 0 & 8 & 5 & 0 \\
Armigeres unidentified sp. & 0 & 0 & 1 & 0 & 0 & 10 & 0 & 0 \\
An. jamesii & 2 & 0 & 0 & 0 & 0 & 2 & 0 & 0 \\
An. aitkenii & 0 & 0 & 0 & 1 & 0 & 0 & 0 & 0 \\
Anopheles unidentified sp. & 2 & 0 & 1 & 0 & 1 & 0 & 3 & 0 \\
\hline Total & $\mathbf{1 1 8}$ & $\mathbf{0}$ & $\mathbf{2 1}$ & $\mathbf{3 6}$ & $\mathbf{2 9}$ & $\mathbf{8 5}$ & $\mathbf{3 9}$ & $\mathbf{0}$ \\
Abundance (as a \%) & $\mathbf{3 5 . 9 7}$ & $\mathbf{0 . 0 0}$ & $\mathbf{6 . 4 0}$ & $\mathbf{1 0 . 9 7}$ & $\mathbf{8 . 8 4}$ & $\mathbf{2 5 . 9 1}$ & $\mathbf{1 1 . 8 9}$ & $\mathbf{0 . 0 0}$ \\
\hline
\end{tabular}

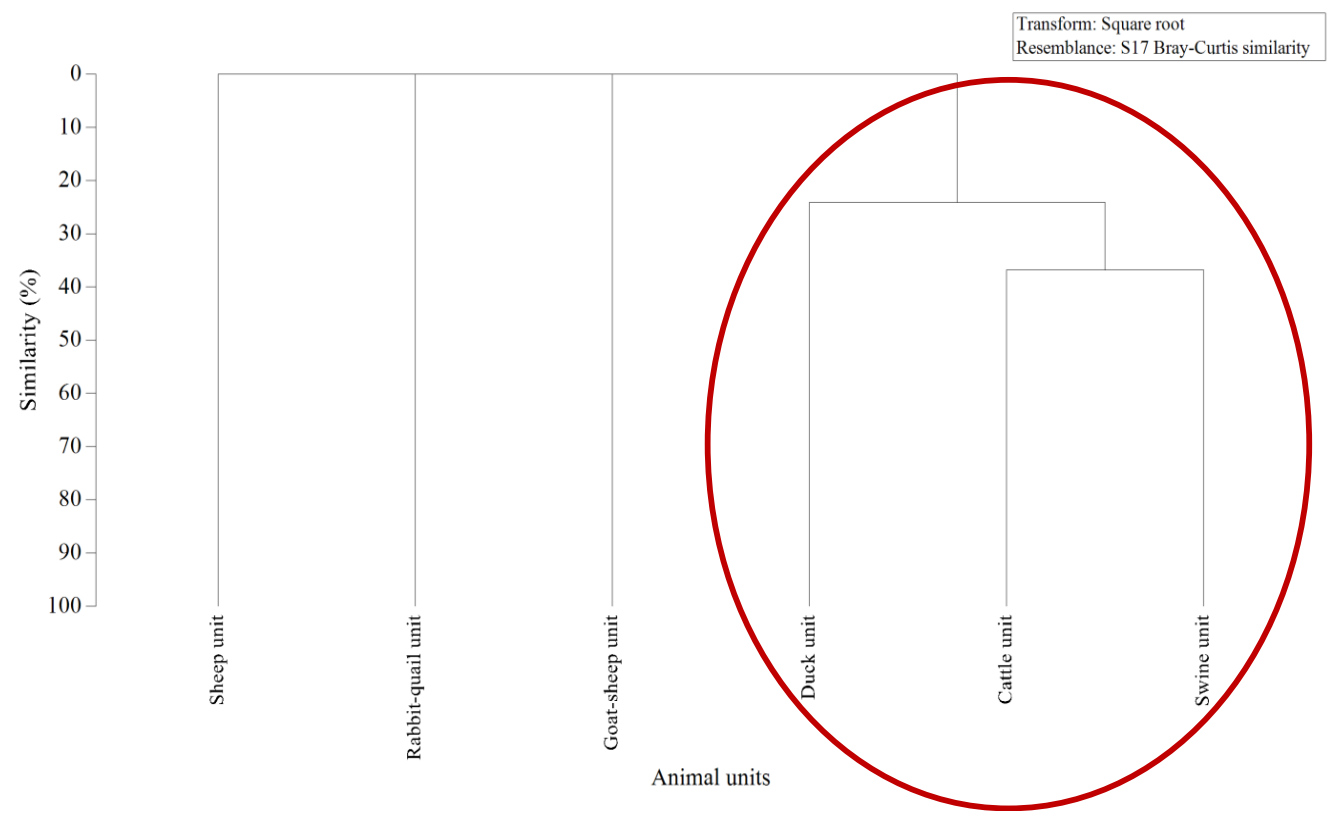

Figure 3: Dendrogram analysis of grouping animal units based on the similarity between species composition of mosquito species.

Dendrogram analysis of grouping animal units based on the similarity in species composition of mosquitoes showed that the mosquito composition of cattle unit and the swine unit was $36.8 \%$ similar (Figure 3). However, similarity in species composition of duck unit to both cattle unit and swine unit was $24.11 \%$. According to the similarity of species composition, cattle, swine and duck unit were included into one main cluster. Other three animal units i.e. sheep, rabbit-quail and goat-sheep were separately clustered in dendrogram analysis.
Pearson's correlation coefficient $(\mathrm{R})$ values showed that there were no significant relationships between the abundance of mosquitoes and the weather parameters tested. However, average temperature $(\mathrm{R}=-0.001, \mathrm{p}$ $=0.999)$ and the wind velocity $(\mathrm{R}=-0.253, \mathrm{p}=0.546)$ showed a weak negative correlation with the mosquito numbers while average rainfall $(\mathrm{R}=0.204, \mathrm{p}=0.629)$ and relative humidity $(\mathrm{R}=0.004, \mathrm{p}=0.992)$ showed a weak positive correlation with the mosquito abundance. 
Although the natural mosquito breeding sites such as tree holes and bromeliads were lacking in the livestock filed station, there were several artificial breeding sites i.e. drainage, metal containers, pieces of PVC pipes and used tires. A total of 135 larvae were collected and 130 emerged as adults and were taxonomically identified. Ochlerotatus pseudotaeniatus larvae were the most abundant accounting to $50.76 \%$ of the total larvae collected. Aedes albopictus was the second most abundant $(33.10 \%)$ followed by Cx. quinquefasciatus (13.84\%) and Cx. pseudovishnui (2.31\%). Positive water samples were dominated by Culex mosquitoes and Ae. albopictus. Larvae of Oc. pseudotaeniatus were collected mainly from clean water.

\section{DISCUSSION}

Findings of this study mainly describe the species composition, abundance, host preferences, host range, preferred weather conditions and breeding sites of mosquito communities in a livestock station. The reported mosquito community of the livestock station comprised of 17different mosquito species of four genera. Culex was the highest abundant mosquito genera followed by Armigeres, Aedes and Anopheles. The Cx. fuscocephala was identified as the dominant mosquito species of the livestock mosquito community.

Out of reported 17 species, 16 species were reported from the cattle unit. Larger body size, relatively less defensive behavior, physiological factors of cattle blood and the host derived signals such as olfactory signals may be the possible causes for the higher attraction of mosquito towards them (Clement, 1999; Zwiebel and Takken, 2004). Also, the cattle release relatively larger amount of carbon dioxide (Santos and Borges, 2015) which could act as a long distance attractant for the mosquitoes.

Although, many previous studies have shown that Culex mosquitoes often feed on birds (Mackay et al., 2010; Farajollahi et al., 2011), the present study showed that less preference of Culex mosquitoes to the bird host when large herbivores like cattle are close by. Higher attraction of $C x$. tritaeniorhynchus, $C x$. vishnui and $C x$. gelidus to cattle has been previously shown by Mwandawiro et al., (2000). Cx. quinquefasciatus is a well-known Culex species that is attracted to a wide range of hosts including human, cattle, goat, donkey, dogs, cats and birds (Molaei et al., 2007; Muturi et al., 2008). In the present study, this species was collected only from the swine unit $(1.82 \% ; n=$ 6) suggesting that it prefers to feed on swine blood.

The absence of mosquitoes in rabbit-quail, goat-sheep and sheep units indicates that when there is a choice, mosquitoes prefer larger-bodied vertebrates while avoiding small vertebrates including birds. These findings are consistent with the reports of previous studies (Chandler et al., 1975, 1976; Gad et al., 1999; Seyoum et al., 2002; Tchouassi et al., 2016). Availability and defensive behaviors of host, nutritional value of the blood meal and the energy cost of digestion of the blood have been identified as the determinant factors that influence the host preferences (Lyimo and Ferguson, 2009; Santos and Borges, 2015).
Dendrogram analysis grouped the animals according to the host preference range of mosquitoes. The prominent cluster included mosquitoes that share cattle and swine units. The duck unit was separated from the main cluster and Cx. hutchinsoni and Ae. albopictus were recorded only from the duck unit. Goat-sheep unit, sheep unit and rabbit-quail units were separated from the main cluster due to the absence of mosquitoes in these units during the study period. The four mosquito species that were reported in both cattle and swine unite (main cluster) were diagnosed either with cattle blood or with swine blood according to their place of capture. The results also showed that the tested engorged mosquitoes have taken full blood meals from a single host and did not have any mixed blood meals.

A significant variation in monthly abundance of mosquito species could be observed over the eight month period. The most abundant mosquito species Cx. fuscocephala, $C x$. gelidus, Cx. pseudovishnui, and Cx. tritaeniorhynchus were more or less evenly distributed throughout the study period except for the months of January and July. The highest number of mosquitoes was recorded during the months of December and May while some of the species reported only during specific months. The diversity indices showed relatively higher values during April and May, and in December where south-west monsoon and north-east monsoon occur respectively. The study reported noticeable fluctuations in the monthly abundance of mosquitoes in the livestock station only for an eight month period. However, further studies are undergoing to better understand the periodic fluctuations and the effect of changing weather pattern and farm environment on the abundance, diversity and the feeding pattern of livestock mosquitoes.

Correlation analysis did not show strong relationships between the mosquito abundance and weather variables. The wind speed affects the abundance pattern of mosquitoes and it has been reported that the wind velocity more than $3 \mathrm{~km} / \mathrm{h}$ can considerably reduce the host seeking flight behavior of mosquitoes (Service, 1980). Many studies have shown influence of rainfall on mosquito abundance (Roiz et al., 2014; Abiodun et al., 2016; Valdez et al., 2017). However, further studies are needed for a better understanding of the population dynamics of these species in the livestock stations in Sri Lanka.

Availability of breeding sites is one of the contributing factors for abundance, diversity and the population dynamics of mosquitoes in a particular habitat (Vezzani et al., 2004). We did not observe noticeable variations of the available breeding sites in the study location. Although the highest number of larvae was from Oc. pseudotaeniatus, no adults could be collected from this species. The activity pattern and the biting behavior of this mosquito species are not well known. The time of our collection may not be the active time period of this species or Oc. pseudotaeniatus may not be attracted to any of these hosts. Ae. albopictus is a well-known day time biter and the low abundance of this species in night collections can be expected. 


\section{CONCLUSION}

The study reveals that the mosquito community of the livestock field station comprised of many generalists that feed on a wide range of host species including humans. The most abundant mosquito species such as $C x$. tritaeniorhynchus, $C x$. vishnui, $C x$. gelidus and $C x$. fuscocephalaare are well known for transmitting diseases like Japanese encephalitis. These mosquitoes can act as bridge vectors of diseases to both humans and the livestock because of the availability of both potential vectors and domestic animals which can act as reservoir/ amplifying hosts in the vicinity of human settlements.

\section{ACKNOWLEDGEMENTS}

We are thankful to academic and non-academic staff of the faculty of Agriculture and the Livestock Filed Station at Uda Peradeniya for their assistance during mosquito sampling. We are also thankful to Mr. Dilan Chathuranga, Mr. Nalaka Nugapola, and Miss Thisari Gunathilaka for their assistance in the field as well as in the lab. This work was supported by the University Grant No: URG /2016/51/S given to the corresponding author.

\section{REFERENCES}

Abiodun, G. J., Okosun, K. O., Witbooi, P. and Maharaj, R. (2016). Modelling the influence of temperature and rainfall on the population dynamics of Anopheles arabiensis. Malaria Journal 15:364.

Abrams, P. A. (2006). The prerequisites for and likelihood of generalist-specialist coexistence. The American Naturalist 167:329-342.

Amerasinghe, F. P. (1990). A guide to the identification of the Anopheline mosquitoes (Diptera: Culicidae) of Sri Lanka. I. Adult females. Ceylon Journal of Science, Biological Sciences 20:1-16.

Amerasinghe, F. P. (1995). Illustrated keys to the genera of mosquitoes (Diptera: Culicidae) in Sri Lanka. Journal of the National Science Foundation of Sri Lanka. 23: 183-211.

Barraud, P. J. (1934). The Fauna British India, including Ceylon and Burma. 5. London: Taylor \& Francis.

Bhattacharya, S., Basu, P. and Sajal Bhattacharya, C. (2016). The southern house mosquito, Culex quinquefasciatus: profile of a smart vector. Journal of Entomology and Zoology Studies 4:73-81.

Clements, A. N. (1999). The biology of mosquitoes: Sensory, Reception, and Behavior. London. UK: Chapman \& Hall.

Chandler, J. A., Boreham, P. F. L., Highton, R. B. and Hill, M. N. (1975). A study of the host selection patterns of the mosquitoes of the Kisumu area of Kenya. Transactions of the Royal Society of Tropical Medicine and Hygiene 69:415-425.

Chandler, J. A., Highton, R. B. and Boreham, P. F. L. (1976). Studies on some Ornithophilic mosquitoes (Diptera, Culicidae) of the Kano Plain, Kenya. Bulletin of Entomological Research 66:133-143.

Farajollahi, A., Crans, W. J., Bryant, P., Wolf, B., Burkhalter, K. L., Godsey, M. S., Aspen, S. E. and Nasci, R. S.
(2005). Detection of West Nile viral RNA from an overwintering pool of Culex pipenspipiens (Diptera: Culicidae) in New Jersey, 2003. Journal of Medical Entomology 42:490-494.

Gaffigan, T. and Pecor, J. (1997).Collecting, rearing, mounting and shipping mosquitoes. Available from: wrbu. si. edu/Techniques. html.

Gad, A. M., Farid, H. A., Ramzy, R. R., Riad, M. B., Presley, S. M., Cope, S. E., Hassan, M. M. and Hassan, A. N. (1999). Host feeding of mosquitoes (Diptera: Culicidae) associated with the recurrence of Rift Valley fever in Egypt. Journal of Medical Entomology 36: 709-714.

Garcia-Rejon, J. E., Blitvich, B. J., Farfan-Ale, J. A., Loroño-Pino, M. A., Chim, W. A. C., Flores-Flores, L. F., Rosado-Paredes, E., Baak-Baak, C., PerezMutul, J., Suarez-Solis, V. and Fernandez-Salas, I. (2010). Host-feeding preference of the mosquito, Culex quinquefasciatus, in Yucatan State, Mexico. Journal of Insect Science 10:1-12.

Gingrich, J. B. and Williams, G. M. (2005). Host-feeding patterns of suspected West Nile virus mosquito vectors in Delaware, 2001-2002. Journal of the American Mosquito Control Association 21:194-200.

Kent, R. J. and Norris, D. E. (2005). Identification of mammalian blood meals in mosquitoes by a multiplexed polymerase chain reaction targeting cytochrome $\mathrm{B}$. The American Journal of Tropical Medicine and Hygiene 73:336-342.

Lehane, M. J. (2005). The biology of blood-sucking in insects. $2^{\text {nd }}$ Ed. Cambridge, UK: Cambridge University Press.

Lima, J. B. P., Rosa-Freitas, M. G., Rodovalho, C. M., Santos, F. and Lourenço-de-Oliveira, R. (2014). Is there an efficient trap or collection method for sampling Anopheles darlingi and other malaria vectors that can describe the essential parameters affecting transmission dynamics as effectively as human landing catches?-A Review. Memorias do InstitutoOswaldo Cruz 109:685705 .

Livak, K. J. (1984). Organization and mapping of a sequence on the Drosophila melanogaster X and Y chromosomes that is transcribed during spermatogenesis. Genetics 107: 611-634.

Mackay, A. J., Kramer, W. L., Meece, J. K., Brumfield, R. T. and Foil, L. D. (2010). Host feeding patterns of Culex mosquitoes (Diptera: Culicidae) in east batonrouge parish, Louisiana. Journal of Medical Entomology 47:238-248.

Lyimo, I. N. and Ferguson, H. M. (2009). Ecological and evolutionary determinants of host species choice in mosquito vectors. Trends in Parasitology 25:189-196.

Molaei, G., Andreadis, T. G., Armstrong, P. M., Anderson, J. F. and Vossbrinck, C. R. (2006).Host feeding patterns of Culex mosquitoes and West Nile virus transmission, northeastern United States. Emerging Infectious Diseases 12:468-474.

Molaei, G., Andreadis, T. G., Armstrong, P. M., BuenoJr, R., Dennett, J. A., Real, S. V., Sargent, C., Bala, A., Randle, Y., Guzman, H. and da Rosa, A. T. (2007). Host feeding pattern of Culex quinquefasciatus (Diptera: 
Culicidae) and its role in transmission of West Nile virus in Harris County, Texas. The American Journal of Tropical Medicine and Hygiene 77:73-81.

Muturi, E. J., Muriu, S., Shililu, J., Mwangangi, J. M., Jacob, B. G., Mbogo, C., Githure, J. and Novak, R. J. (2008). Blood-feeding patterns of Culex quinquefasciatus and other culicines and implications for disease transmission in Mwea rice scheme, Kenya. Parasitology Research 102:1329.

Mwandawiro, C., Boots, M., Tuno, N., Suwonkerd, W., Tsuda, Y. and Takagi, M. (2000). Heterogeneity in the host preference of Japanese encephalitis vectors in Chiang Mai, northern Thailand. Transactions of the Royal Society of Tropical Medicine and Hygiene 94: 238-242.

Peiris, J.S.M., Amerasinghe, P.H., Amerasinghe, F.P., Calisher, C.H., Perera, L.P., Arunagiri, C.K., Munasinghe, N.B., Karunaratne, S.H.P.P. and Weligama, N. (1994). Virus isolation from mosquitoes collected in Sri Lanka. American Journal of Tropical Medicine and Hygiene 51:154-161.

Pyke, G. H., Pulliam, H. R. and Charnov, E. L. (1977). Optimal foraging: a selective review of theory and tests. The Quarterly Review of Biology.52: 137-154.Roiz, D., Figuerola, J., Soriguer, R. and Ruiz, S. (2014). Climatic effects on mosquito abundance in Mediterranean wetlands. Parasites \& Vectors. 7:347-353.

Santos, C. F. and Borges, M. (2015). Impact of livestock on a mosquito community (Diptera: Culicidae) in a Brazilian tropical dry forest. Revista da Sociedade Brasileira de Medicina Tropical 48:474-478.

Service, M. W. (1980). Effects of wind on the behaviour and distribution of mosquitoes and blackflies. International Journal of Biometeorology 24:347-353.

Seyoum, A., Balcha, F., Balkew, M., Ali, A. and GebreMichael, T. (2002). Impact of cattle keeping on human biting rate of Anopheline mosquitoes and malaria transmission around Ziway, Ethiopia. East African Medical Journal 79:485-490.
Takken, W. andVerhulst, N. O. (2013). Host preferences of blood-feeding mosquitoes. Annual Review of Entomology 58:433-453.

Tchouassi, D. P., Okiro, R. O., Sang, R., Cohnstaedt, L. W., McVey, D. S. and Torto, B. (2016). Mosquito host choices on livestock amplifiers of Rift Valley fever virus in Kenya. Parasites \& Vectors 9:184.

Tempelis, G. H. and Washino, R. K. (1967). Host-feeding patterns of Culex tarsalis in the Sacramento Valley, California, with notes on other species. Journal of Medical Entomology 4:315-318.

Tempelis, C. H., Reeves, W. C. and Nelson, R. L. (1976). Species identification of blood meals from Culex tarsalis that had fed on passeriform birds. The American Journal of Tropical Medicine and Hygiene 25:744-746.

Thiemann, T. C., Wheeler, S. S., Barker, C. M. and Reisen, W. K. (2011). Mosquito host selection varies seasonally with host availability and mosquito density. PLoS Neglected Tropical Diseases 5:1452.

Valdez, L. D., Sibona, G. J., Diaz, L. A., Contigiani, M. S. and Condat, C. A. (2017). Effects of rainfall on Culex mosquito population dynamics. Journal of Theoretical Biology. 421:28-38.

Vezzani, D., Velázquez, S.M. and Schweigmann, N. (2004). Seasonal pattern of abundance of Aedes aegypti (Diptera: Culicidae) in Buenos Aires city, Argentina. Memórias do Instituto Oswaldo Cruz 99:351-356.

WHO (2017). Rift Valley fever. [Online] Available from: http://www.who.int/mediacentre/factsheets/fs207/en/. [Accessed on: $3^{\text {rd }}$ December 2017].

Zwiebel, L. J. and Takken, W. (2004). Olfactory regulation of mosquito-host interactions. Insect Biochemistry and Molecular Biology 34:645-652. 